\title{
Well, being a dentist
}

\author{
Stephen Hancocks OBE \\ Editor in Chief
}

The $B D J$ Upfront section includes editorials, letters, news, book reviews and interviews. Please direct your correspondence to the News Editor,

Kate Quinlan at k.quinlan@nature.com. Press releases or articles may be edited, and should include a colour photograph if possible.

I am not a millennial: ok. So for me, the first time I travelled in an airplane was after I qualified as a dentist. I know, how last century is that? But when I did I was shocked at the safety briefing. 'In the event of a loss in pressure, oxygen masks will drop from the panel above your head. Be sure to attach your own mask before helping others.' For the previous five or more years everything I had been taught had been about caring for others first, about selflessness and yet here was an established airline dashing aside such ethical considerations. I cautiously glanced sideways at the adjacent passenger. In fairness he looked as if this wasn't his first flight and I didn't anticipate that he would need my help in an emergency. Probably the opposite.

With reflection, the advice is pure common sense, one has to save oneself before one can even attempt to save, or help, another person. If you're incapacitated or dead you're of little or no use to anyone. It is a matter of biology too. As J Z Young asks far more cogently in his master work An introduction to the study of man, 'who repairs the repairer of the repairer?'. ${ }^{1}$ Yet there remains a lingering doubt in our minds doesn't there? Even the General Dental Council's (GDC) Standards for the dental team states that 'there are nine principles registered dental professionals must keep to at all times. As a GDC registrant you must: put patients' interests first. ${ }^{2}$

The reason for cogitating on this is the matter of our own health and well-being as given focus in a paper in this issue by Colonio Salazar et al. ${ }^{3}$ Reviewing two decades of literature, the authors aimed to identify key determinants of health and well-being amongst dentists working in the UK. The studies examined health (physical, psychological and emotional), personal (eg anxiety, happiness, worthwhileness, and life satisfaction), or job well-being (eg job satisfaction, morale, motivation, work engagement, and burnout).
Although research, because of the time it takes to organise, complete and publish will always lag behind real world intelligence or current sensitivities, the findings of this review do seem to accord with the trend that most of us might recognise in ourselves and amongst our colleagues. Overall, dentists' health and personal well-being appeared to be 'good'; however, recent evidence suggests that we are becoming more concerned about these issues. Also, in relation to job well-being, whilst for the most part dentists report high levels of job and career satisfaction and low levels of burnout, we have recently reported being less positive and more at risk of suffering from stress than indicated in findings from research two decades ago. engagement, and lower levels of burnout and stress. Also, significantly in my view and based on content we have published here in recent times, they note the emergence of regulation as a determinant of poor health and well-being which may be associated with contemporary culture. This is specifically in association with a tightening of professional regulation and patient-concerns being given more weight by the regulator.

Whether you regard yourself as a repairer or one who needs repairing, an oxygen-mask fitter or recipient of the same it behoves us all to pay attention to our individual health and well-being, to not be ashamed of this approach and to not undermine it in relation to the care of our patients. Policy makers,

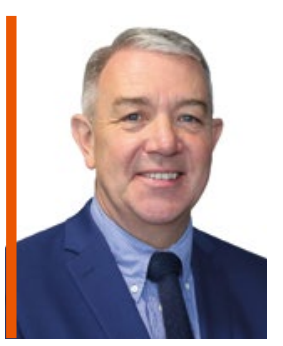

\section{'One has to save oneself before one can even attempt to save, or help, another person'}

While the study cautions about drawing too many conclusions given the quality of research surveyed, it does spotlight these more recent concerns as well as the usual suggestion for the need for further well-designed and conducted studies. The authors give a very good overview of the potential factors affecting us with our various personal characteristics - age, experience, role, specialty and so forth - which emphasises the wide range of considerations and stresses placed upon us in the immediate and longerterm future. Two elements that they identify will hardly surprise us. In terms of healthcare systems, those dentists mainly working outside the NHS appear to experience higher levels of personal well-being, career and job satisfaction, motivation and morale and work healthcare organisations and dental practices can all help in fostering good working environments but individual assessment and action is paramount. There is no question that without our help patients' oral health will suffer and without our own good health we simply cannot offer an optimum service. It is time to look after ourselves too.

\section{References}

1. Young J Z. An introduction to the study of man. Oxford University Press, 1985.

2. GDC. Standards for the dental team. 2013. Available at https://www.gdc-uk.org/api/files/NEW\%20 Standards\%20for\%20the\%20Dental\%20Team.pdf (accessed July 2019).

3. Colonio Salazar F B, Sipiyaruk K, White S, Gallagher J E. Key determinants of health and well-being of dentists within the UK - A rapid review of over two decades of research. Br Dent J 2019; 227: 127-136.

DOl: $10.1038 / 541415-019-0573-3$ 